\title{
Salt tolerance involved candidate genes in rice: an integrative meta-analysis approach
}

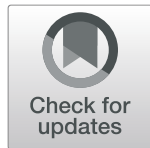

Raheleh Mirdar Mansuri ${ }^{1,2}$, Zahra-Sadat Shobbar ${ }^{1 *}$ (D, Nadali Babaeian Jelodar ${ }^{2}$, Mohammadreza Ghaffari ${ }^{1}$, Seyed Mahdi Mohammadi ${ }^{1}$ and Parisa Daryani ${ }^{1}$

\begin{abstract}
Background: Salinity, as one of the main abiotic stresses, critically threatens growth and fertility of main food crops including rice in the world. To get insight into the molecular mechanisms by which tolerant genotypes responds to the salinity stress, we propose an integrative meta-analysis approach to find the key genes involved in salinity tolerance. Herein, a genome-wide meta-analysis, using microarray and RNA-seq data was conducted which resulted in the identification of differentially expressed genes (DEGs) under salinity stress at tolerant rice genotypes. DEGs were then confirmed by meta-QTL analysis and literature review.
\end{abstract}

Results: A total of 3449 DEGs were detected in 46 meta-QTL positions, among which 1286, 86, 1729 and 348 DEGs were observed in root, shoot, seedling, and leaves tissues, respectively. Moreover, functional annotation of DEGs located in the meta-QTLs suggested some involved biological processes (e.g., ion transport, regulation of transcription, cell wall organization and modification as well as response to stress) and molecular function terms (e.g., transporter activity, transcription factor activity and oxidoreductase activity). Remarkably, 23 potential candidate genes were detected in Saltol and hotspot-regions overlying original QTLs for both yield components and ion homeostasis traits; among which, there were many unreported salinity-responsive genes. Some promising candidate genes were detected such as pectinesterase, peroxidase, transcription regulator, high-affinity potassium transporter, cell wall organization, protein serine/threonine phosphatase, and CBS domain cotaining protein.

Conclusions: The obtained results indicated that, the salt tolerant genotypes use qualified mechanisms particularly in sensing and signalling of the salt stress, regulation of transcription, ionic homeostasis, and Reactive Oxygen Species (ROS) scavenging in response to the salt stress.

Keywords: Meta- analysis, RNA-seq, Microarray, QTLs, Salinity stress, Oryza sativa

\section{Background}

Currently, rice ranks as the most important food crop in the world before wheat and maize supplying a major source of calorie for more than 3.5 billion people all over the world [1, 2]. However, rice is classified as a very sensitive crop to salinity in both seedling and reproductive stages, while excess salt in soil is one of the most

\footnotetext{
* Correspondence: shobbar@abrii.ac.ir

'Department of Systems Biology, Agricultural Biotechnology Research Institute of Iran (ABRII), Agricultural Research, Education and Extension Organization (AREEO), PO Box 31535-1897, Karaj, Iran

Full list of author information is available at the end of the article
}

widespread abiotic stresses in Asia and some river deltas in Europe [3, 4]. Salinity challenge at the seedling stage causes the growth arrest or death of rice plant, that reduces significantly the yield $[5,6]$; therefore, increasing the salinity tolerance at the seedling stage would be effective to improve the environmental adaptation and yield maintenance in rice. It is necessary to understand the mechanisms underlying the salinity stress tolerance because of increasing the population, limited arable land, and climate changes that can provide us a better perspective regarding how to manage the increasing demand for high-yielding rice [2, 7]. Salinity tolerance is a

(c) The Author(s). 2020 Open Access This article is licensed under a Creative Commons Attribution 4.0 International License, which permits use, sharing, adaptation, distribution and reproduction in any medium or format, as long as you give appropriate credit to the original author(s) and the source, provide a link to the Creative Commons licence, and indicate if changes were made. The images or other third party material in this article are included in the article's Creative Commons licence, unless indicated otherwise in a credit line to the material. If material is not included in the article's Creative Commons licence and your intended use is not permitted by statutory regulation or exceeds the permitted use, you will need to obtain permission directly from the copyright holder. To view a copy of this licence, visit http://creativecommons.org/licenses/by/4.0/ The Creative Commons Public Domain Dedication waiver (http://creativecommons.org/publicdomain/zero/1.0/) applies to the data made available in this article, unless otherwise stated in a credit line to the data. 
complicated trait both genetically and physiologically [8]. Rice, as a well-studied model organism, is particularly rewarding to investigate the salinity stress responses [7]. Many QTLs have been eventually identified in the rice breeding programs [9-16], including a major locus on chromosome 1, namely Saltol, involved in $\mathrm{Na} / \mathrm{K}$ homeostasis derived from Pokkali and SKC1 (OsHKT1; 5) from Nona Bokra [17]. Isolation of the identified QTLs related to salt tolerance can be highly beneficial to improve the global agriculture and food security but it is also a challenging task [18]. Although, many QTLs have been found but there is still limited knowledge regarding the salinity tolerance-related gene networks in rice. Technologies such as microarray and gene expression profiling based on sequencing approaches accelerate the progress toward a comprehensive understanding of the genetic mechanisms related to responses to environmental stresses $[19,20]$. Fast advances and decreased price of high-throughput sequencing technology have led to extensive application of RNA sequencing in various species in the recent years [21]. Therefore, many differentially expressed genes (DEGs) have been identified among the contrasting samples through mentioned technologies. Researchers have recently used an integration of DEGs and QTLs as a confident method to identify the potential candidate genes [22]. Currently, a great and varied set of genomic data has become publicly available; subsequently, a combination of numerous accessible data can rise the consistency and generalizability of the results. Combining the results obtained from the independent but associated studies is called "meta-analysis (MA)"; thus researchers can obtain more exact estimation regarding the differential gene expression by increasing the statistical power in MA [23, 24]. Breeding by introgression of the identified QTLs is restricted owing to the conflict of QTLs in different genetic backgrounds and environments [25]; while meta- QTL analysis suggests a chance to use QTL data from various mapping populations with diverse genetic backgrounds to detect the accurate position of the QTLs [26]. Several studies have identified the accurate meta-QTLs of with various traits for mining the candidate genes in rice and other crop plants [26-30]. However, an integrative meta-analysis approach was employed in this study that resulted in finding several promising genes involved in salinity tolerance, among which, some of the important genes/gene families with sufficient evidence are listed and discussed later to support their candidacy in the rice. All data produced in the previous studies were used to identify the rice candidate genes related to salt tolerance and then, the candidate genes were confirmed using the meta-analysis. Findings of this study provide valuable information on the genes and pathways involved in salinity tolerance in rice.

\section{Results}

Salinity tolerance associated Meta-QTLs in rice

A total of 265 QTLs related to 32 traits were collected in this study using the Simple Sequence Repeats (SSR) markers (Table S1, S2) among which, 126 and 139 QTLs were selected for further analysis in normal and salinity conditions (Table S3). Most of the QTLs belonged to the salinity tolerance score (STS) (27 QTLs), shoot potassium concentration (KS) (26 QTLs), shoot sodium concentration (NS) (21 QTLs), chlorophyll content (CHL)(19 QTLs) and shoot dry weight (DSW) traits (19 QTLs) (Fig.S1). In contrast, the rare QTLs belonged to the number of sterile spikelets (NSS) [20], dead seedling rate (DSR), leaf potassium concentration (KLV), reduction of seedling height (RSH) and reduction of leaf area (RLA) traits (Fig.S1). The highest number of QTLs were observed on chromosome 1 (37 QTLs) and 2 (36 QTLs) followed by chromosome 7 (29 QTLs), while chromosome 8 (12 QTLs) and 11 (12 QTLs) had the lowest number of QTLs (Fig.S2). The phenotypic variance described by the original QTLs was different from 0.7 to $33.25 \%$ and the confidence interval (CI) of markers was different from 0.99 to $84.36 \mathrm{cM}$ (Table S3). After the integration of all the collected QTLs on the consensus map, 46 meta-QTLs were identified in 12 chromosome of rice (Fig. 1). There were meta-QTLs with a CI of 95\% based on the lowest Akaike information criterion (AIC) values. Remarkably, second meta-QTLs on Chr7: MQTL2, Chr2: M-QTL2, and Chr1: M-QTL2 included the highest number of initial QTLs $(17,16$ and 12 , respectively), which covered a relatively narrow CI $(4.78,1.82$ and $2.84 \mathrm{cM}$, respectively) (Table S4). These meta-QTLs support the important traits; for example, ratio of the shoot sodium and potassium concentration (NKS), number of fertile spikelets (NFS), root length (RTL), and chlorophyll content (Table S4). Chr12: M-QTL4, Chr 9: M-QTL3 and Chr3: M-QTL2 had the highest mean percentage of phenotypic variation $\left(R^{2}\right)$, which can be considered as the main effective QTLs for the involved traits (Table S4). A total of 9366 genes were detected in 46 meta-QTL positions, among which, Chr8: M-QTL2 contained the highest number of genes (868 genes); while, Chr12: M-QTL2 contained the lowest number of genes (14 genes) (Table S4). Moreover, the proportion of functionally characterized annotated genes (27\%) is actually limited compared to the about $73 \%$ of unannotated genes with allocated putative functions. It is intersting to note that, 81 genes were identified on Chr1: M-QTL2 which were located in Saltol region.

\section{Expression profiling analyses in the salinity tolerant genotypes of rice}

The DEGs were identified under salinity stress compared to control conditions in the salinity tolerant genotypes. 


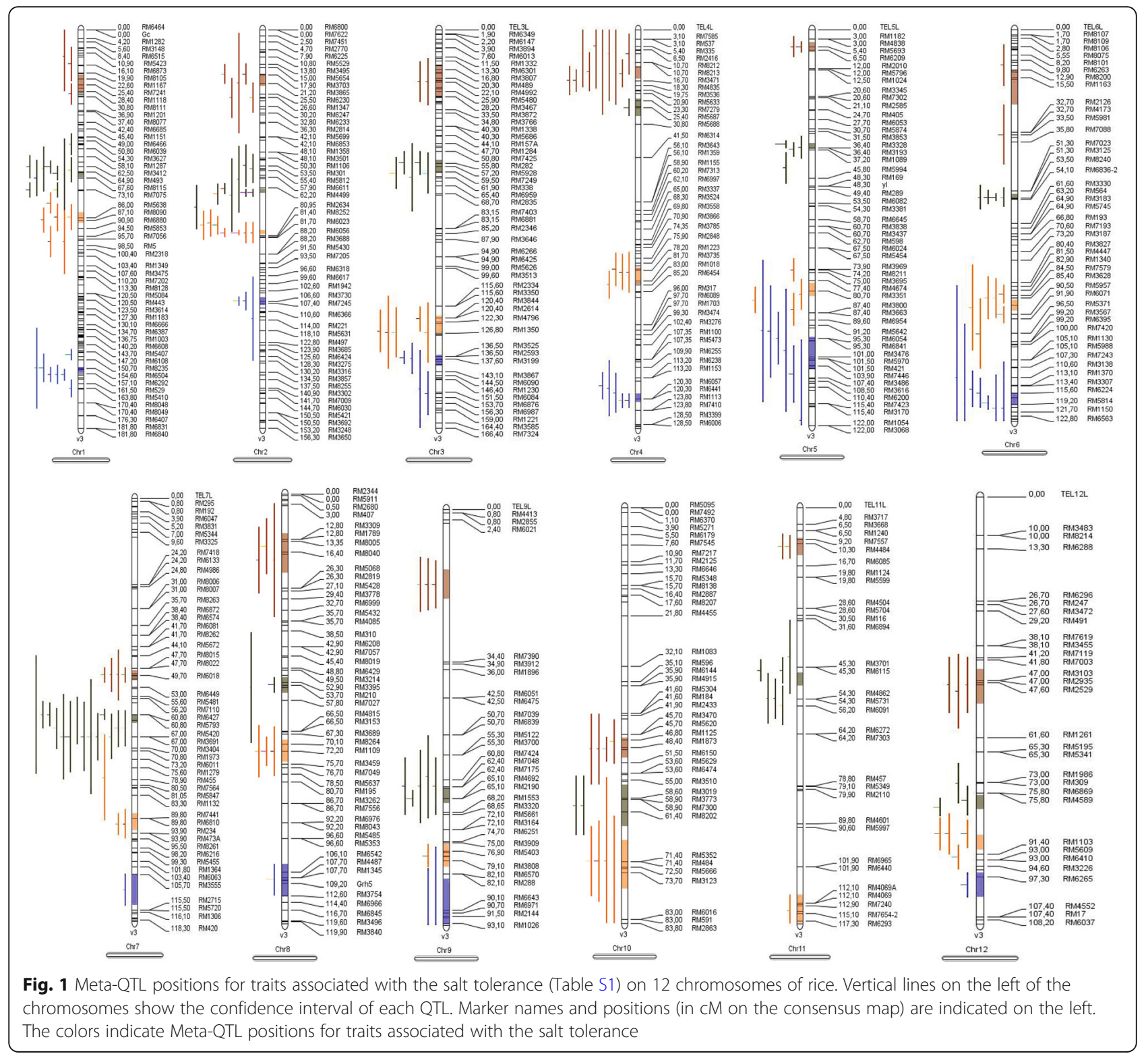

A total of 1714 DEGs were observed in the roots of FL478 as a salinity tolerant genotype, among which, 927 and 787 were up- and down-regulated in the salinity conditions [31]. DEGs from multiple RNA-seq datasets were combined and the DEGs were classified into root, shoot, seedling, and leaves to have a deeper understanding about the salt responsive genes in the salinity tolerant rice genotypes. A total of 3030, 396, 703 and 723 DEGs were merely identified in root, shoot, seedling and leaves, respectively (Fig.S3). Also, raw microarray data from nine independent experiments were downloaded (Table S5) and analyzed uniformly. Microarray metaanalysis suggested 11,694 DEGs, among which, 4121, 13, 6247 and 1199 DEGs were exclusively expressed in root, shoot, seedling and leaves, respectively (Fig.S4). In addition, a total of 4763 and 5862 DEGs were merely up- and down-regulated, respectively, in the salinity tolerant genotypes.

\section{Integration of DEGs from two Meta-analysis approaches} Identified DEGs in both RNA-Seq and microarray metaanalysis were combined to confirm the consistency of the obtained results. A list of overlapping DEGs were detected in four tissues, separately after removing all the duplicate genes.

Comparative transcriptome analysis indicated that 227, 2, 311, and 84 DEGs were commonly detected by the RNA-Seq and microarray respectively in root, shoot, seedling, and leaves tissues (Fig. 2). A total of 4255 and 10,980 DEGs were merley identified by the 


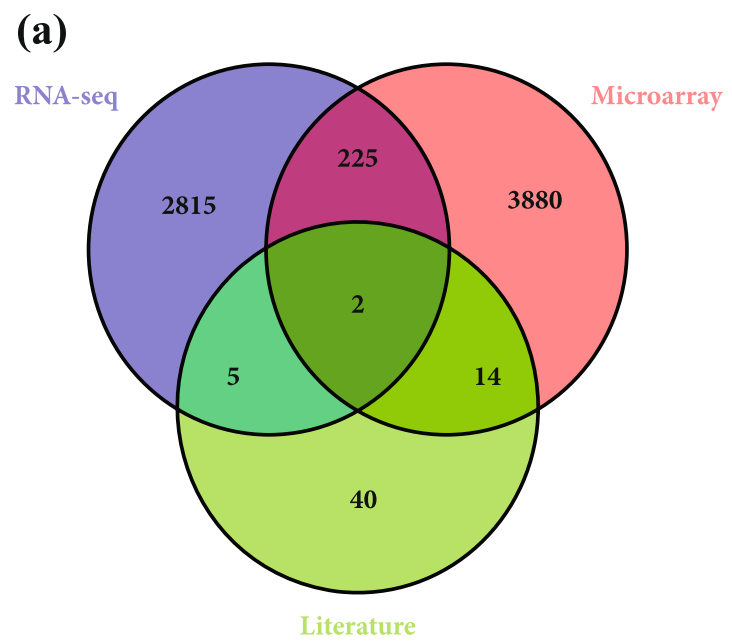

(c)

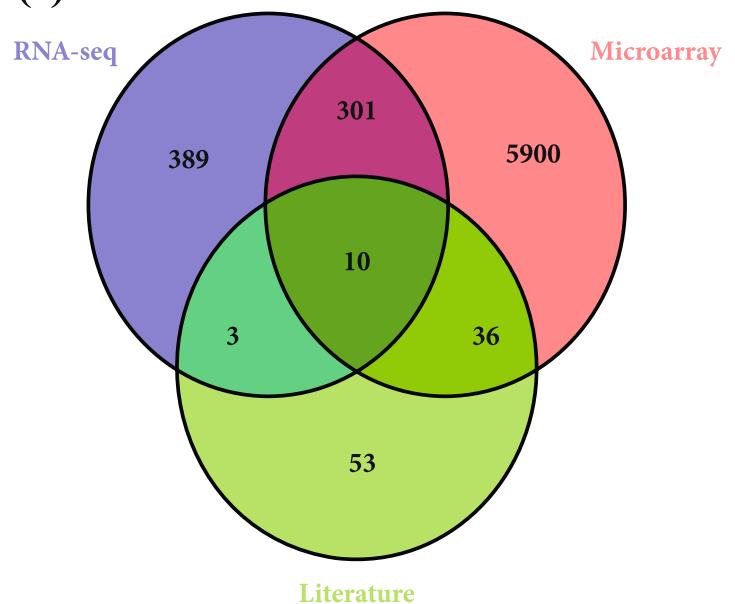

(b)

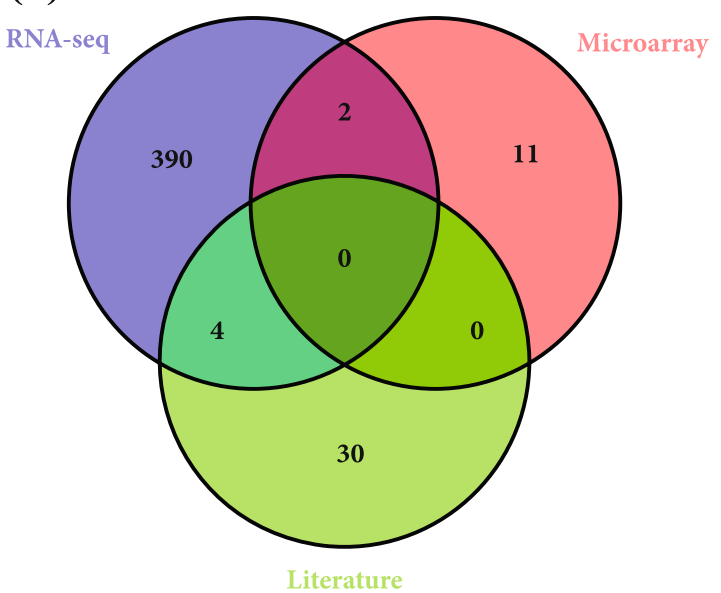

(d)

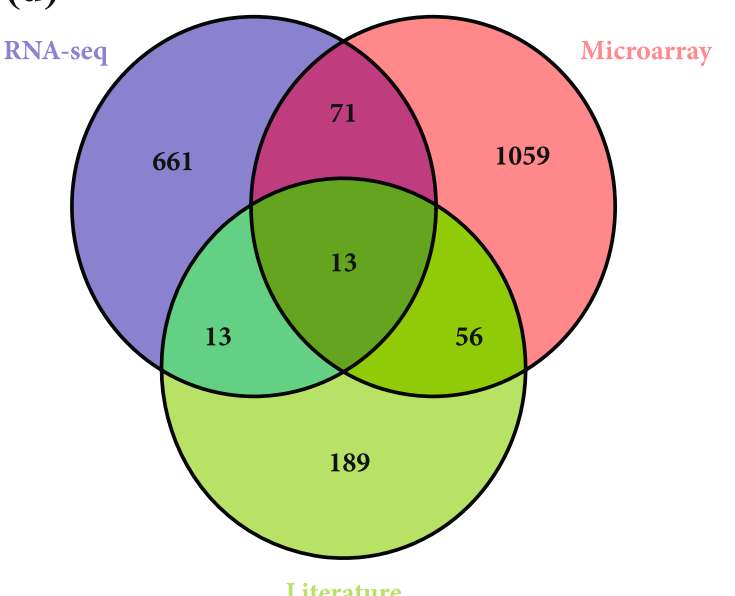

Fig. 2 The results of comparison between differentially expressed genes under salt stress conditions in the tolerant genotypes revealed by RNASeq and microarray data analysis, or through literature review in (a) root, (b) shoot, (c) seedling and (d) leaves

RNA-Seq and microarray meta-analysis, while only 156 DEGs were previously reported in the literature (Fig. 2).

\section{Detection of the DEGs in the meta-QTL positions}

There were a total of $1345,86,1729$, and 552 DEGs in the meta-QTL positions in root, shoot, seedling and leaves, respectively (Fig. 3). Among the identified DEGs in the meta-QTL positions, 664 and 2359 DEGs were identified by the RNA-Seq and microarray meta-analysis, respectively while, only 82 DEGs located in the metaQTL positions were previously reported in the literature (Fig. 3).

\section{Functional annotation of DEGs located in the meta-QTL positions}

Gene ontology enrichment analysis was performed to determine the biological roles of the DEGs located in the meta-QTL positions. Carbohydrate metabolic process, regulation of cellular process, regulation of transcription, response to stress and regulation of nitrogen compound metabolic process were indicated as dominant terms in the biological processes (BP) (Fig.S5). Moreover, some BP terms including regulation of transcription, inorganic anion transport, anion transport, ion transport as well as regulation of gene expression, cell wall organization and modification were significantly enriched (Fig.S5). The most significant over-represented molecular function (MF) terms were nucleotide binding, ATP binding, anion transmembrane transporter activity, inorganic anion transmembrane transporter activity, transcription factor activity and oxidoreductase activity (Fig.S5). In terms of cellular component (CC) ontology, the most significant enriched terms were intrinsic to membrane and integral to membrane (Fig.S5). 


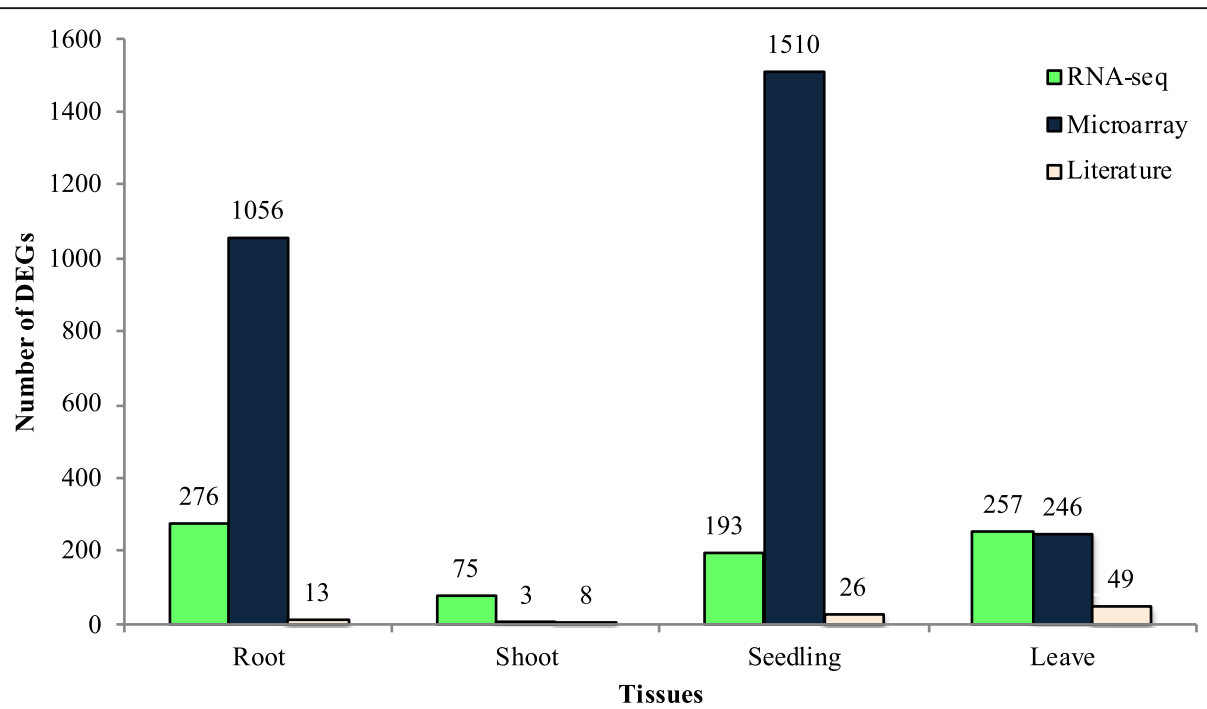

Fig. 3 The number of differentially expressed genes identified by RNA-Seq and microarray data analysis, or through literature review, which are located on the meta-QTL positions in each tissue (roots, shoots, seedlings, and leaves)

\section{Mining the potential candidate genes in the meta-QTL positions}

Exploring the meta-QTL regions for the common genes were resulted in finding 60 potential candidate genes in the root (Table S6), among which, only four genes were previously reported associated to the salinity response. Remarkably, LOC_Os01g20980.1 (coding Pectinesterase) was found in Chr1: M-QTL2 located in Saltol region (Table S6). Ion homeostasis related QTLs were also found in Chr1: M-QTL2 which controling the KLV, NS, NKS, KS and RN traits (Table S4). Overall, identified potential candidate genes were classified into several terms in the root tissue, for example, transcription factor (e.g., TIFY, GRAS, HOX, WRKY and MYB family), signaling (e.g., OsWAK125, pectinesterase,OsMKK1, and CHIT15), transporter (e.g., OsHKT1 and some genes coding transmembrane transport and anion transporter) and some other functions (e.g., NUDIX family, genes coding the aspartic protease) (Table S6).

Four genes in meta-regions on Chr2, 3, and 8 were identified as potential candidate genes in the shoot, as discussed in the literature; for instance, TIP2-1 (LOC_Os02g44080.1) in Chr2: M-QTL4 (Table S6). Chr2: M-QTL4 was integrated with seven initial QTLs controlling RTL and some other related traits (e.g. S, KS, NKS, SIS, and NS) (Table S4). Moreover, two transcription factors (LOC_Os03g08310.1 and LOC_Os08g15050.1) were identified respectively as possible candidate genes in Chr3: M-QTL1 and Chr8: M-QTL2 (Table S6) supporting the root length and photosynthesis related traits, respectively (Table S4). It is interesting to note that, LOC_Os03g08310.1 (coding TIFY11A) was identified as common candidate gene in the root and shoot (Table S6).

Our results indicated 98 potential candidate genes in the seedling including 84 DEGs located in the M-QTLs that were not reported yet. However, 14 genes have been already considered in the literature (Table S6). Functional classification of these potential candidate genes further suggested that they were related to the transcription regulation (e.g., $A P 2$, WRKY, HOX, and GRAM family), signal transduction (e.g., CIPK24, GDSL) and there were some genes with another functions including kinase, phosphatase, and transporter terms under salinity stress in seedling tissue (Table S6). Remarkably, LOC_ Os01g20830.1 (coding a transporter protein) and LOC_ Os01g21144.1 (with unknown function) were found in Saltol region on Chr1: M-QTL2 (Table S6). As well, there were some potential candidate genes in hotspotregions; for example, WRKY70 (LOC_Os05g39720.1) in Chr5: M-QTL4 and PP2C (LOC_Os06g48300.1) in Chr6: M-QTL4 $\left(R^{2}=10.31 \%\right)$ (Table S4, S6). Moreover, some genes were identified as potential candidate genes in Chr2: M-QTL1, Chr8: M-QTL1, Chr10: M-QTL3, and Chr11: M-QTL1; these meta-regions were integrated the importance of the initial QTLs for photosynthesis, straw dry weight, yield components (e.g. QGW, DF and NFS) and RTL traits (Table S4, S6).

Totally, 28 potential candidate genes were identified in the leaves among which, 14 genes were found in the literature. The LOC_Os01g22249.1 (coding the peroxidase) located in Saltol region in Chr1: M-QTL2 was identified as another leading candidate gene. Notably, OsHKT1 (LOC_Os06g48810.1) and PP2C (LOC_ 
Os06g48300.1) were found in the hotspot-regions in Chr6: M-QTL4 (Table S4, S6).

The obtained results indicated that, 20 genes were located on the hotspot-regions containing original QTLs for both yield components and ion homeostasis traits which could be suggested as promising candidate genes (Fig. 4, Table 1). The promising genes were related to the following functions: pectinesterase, peroxidase, transcription regulation, high-affinity potassium transporter, protein serine/threonine phosphatase, cell wall organization and a CBS domain containing gene, among which, there were 2 genes in Saltol region (Table 1).

\section{Validation of differential gene expression using qRT-PCR}

To further validate the potential candidate genes, 15 genes were selected for qRT-PCR in FL478 as a salt tolerant genotype (Fig. 5). The qRT-PCR results were confirmed the outcome of the meta-analysis (Fig.S6).

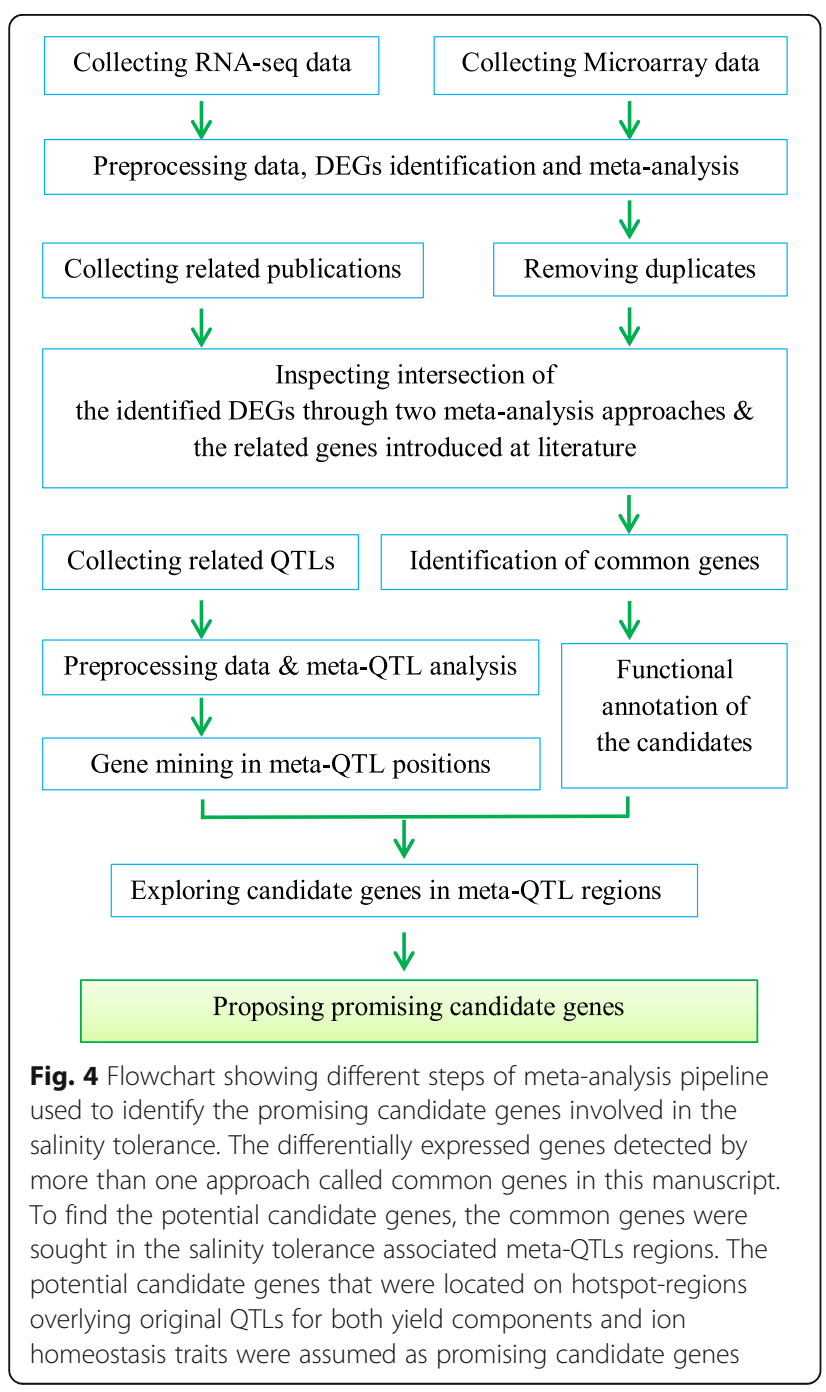

\section{Discussion}

Rice is highly influenced by the salinity stress at seedling and reproductive stages. High salinity concentrations lead to the ionic imbalances, dehydration, osmotic stress, and oxidative damage. Therefore, it is important to identify the most accurate QTLs and the involved candidate genes. Herein, a panel of potential candidate genes both located on the meta-QTL regions and differentially expressed ones in the salinity stress conditions was provided in the tolerant genotypes (Fig. 6).

\section{Sensing and signaling}

Tolerance of the plants against the abiotic stresses including salinity is activated by the complex multicomponent signaling pathways to return the cellular homeostasis and promote the survival [32]. The plant cell wall is one of the first layers for biotic and abiotic stimuli perception, and cell wall remodeling provides a general response mechanism to stresses [33]. Here were several genes coding integral components of membrane and cell wall organization in the hotspot-regions. OsWAK125 was found in Chr12: M-QTL1 and upregulated in the roots (Table S6, Fig. 6), belonging to the wall-associated kinase family and has been mainly investigated as a potential candidate for the cell wall "sensor" $[34,35]$. The Wall Associated Kinases (WAKs) firmly bind to the pectic network of the cell wall, protrude the membrane, and link it to the cytoplasm where a Serine/ Threonine (Ser/Thr) kinase domain is responsible for further signaling $[34,35]$. A drought and salinity responsive class of cell wall-related genes (represented by the pectinesterase) was also found in Saltol region upregulated in the roots (Table S6, Fig. 6). Various crops such as soybean, wheat, and tomato have been shown to have higher levels of pectin remodeling enzymes in tolerant cultivars than susceptible genotypes under salinity and drought stress [33]. Several Ser/Thr phosphatase genes were differentially expressed in the leaves at seedling stage in the hotspot-regions (Table S6). Ser/Thr phosphatases play significant roles in the regulation of the adaptive stress responses and signaling pathways in various crops such as potato, wheat, and rice [36-40].

OsMKK1 in Ch6: M-QTL12 and OsCHIT15 in Chr10: M-QTL3 were also detected, which up-regulated in the roots, and mediating the salinity signaling in rice (Table S6, Fig. 6) [41]. Plant chitinases play an important role in the response to abiotic stress; it has also been reported that hydrolysis of the carbohydrate chains by the chitinases indicates its possible role in signaling or osmotic adjustment functions [34]. Moreover, seven hydrolase coding genes involved in the signaling pathways were among the DEGs located on the meta-QTL regions (Table S6), among which two GDSL-like lipase/ acylhydrolase enzymes in Chr5:M-QTL2 and Chr6:M- 
Table 1 The promising genes associated with salinity tolerance. The differentially expressed genes detected by more than one approach (common genes) and located on meta-QTLs regions overlying original QTLs for both yield components and ion homeostasis traits were assumed as promising candidate genes in this study (the pipeline is presented in Fig. 4)

\begin{tabular}{|c|c|c|c|}
\hline Gene ID & Gene name/ function & $\begin{array}{l}\text { Meta } \\
\text { position }\end{array}$ & $\begin{array}{l}\text { Tissue (Expressed } \\
\text { in) }\end{array}$ \\
\hline $\begin{array}{l}\text { LOC_ } \\
\text { Os01g20980.1 }\end{array}$ & Pectinesterase & $\begin{array}{l}\text { Chr1: M- } \\
\text { QTL2 }\end{array}$ & Root \\
\hline $\begin{array}{l}\text { LOC_ } \\
\text { Os01g22249.1 }\end{array}$ & Peroxidase & $\begin{array}{l}\text { Chr1: M- } \\
\text { QTL2 }\end{array}$ & Leaves \\
\hline $\begin{array}{l}\text { LOC_ } \\
\text { Os02g06410.1 }\end{array}$ & CBS domain containing membrane protein & $\begin{array}{l}\text { Chr2: M- } \\
\text { QTL1 }\end{array}$ & Root \\
\hline $\begin{array}{l}\text { LOC_ } \\
\text { Os02g06640.1 }\end{array}$ & Ubiquitin family protein, putative, expressed & $\begin{array}{l}\text { Chr2:M- } \\
\text { QTL1 }\end{array}$ & Leaves \\
\hline $\begin{array}{l}\text { LOC_ }_{-} \\
\text {Os04g03810.1 }\end{array}$ & OsSub38, Putative Subtilisin homologue, expressed & $\begin{array}{l}\text { Chr4:M- } \\
\text { QTL1 }\end{array}$ & Root \\
\hline $\begin{array}{l}\text { LOC_ } \\
\text { Os04g26870.1 }\end{array}$ & Oxidoreductase, aldo/keto reductase family & $\begin{array}{l}\text { Chr4: M- } \\
\text { QTL2 }\end{array}$ & Seedling \\
\hline $\begin{array}{l}\text { LOC_ } \\
\text { Os04g06910.1 }\end{array}$ & Expressed protein & $\begin{array}{l}\text { Chr4:M- } \\
\text { QTL1 }\end{array}$ & Seedling \\
\hline $\begin{array}{l}\text { LOC_ } \\
\text { Os04g10750.1 }\end{array}$ & Inorganic phosphate transporter & $\begin{array}{l}\text { Chr4:M- } \\
\text { QTL1 }\end{array}$ & Seedling \\
\hline $\begin{array}{l}\text { LOC_ } \\
\text { Os05g42130.1 }\end{array}$ & MOC1,Transcription regulation, GRAS family & $\begin{array}{l}\text { Chr5: M- } \\
\text { QTL4 }\end{array}$ & Root \\
\hline $\begin{array}{l}\text { LOC_ } \\
\text { Os05g39720.1 }\end{array}$ & $\begin{array}{l}\text { WRKY70, Transcription regulation, Negative regulator of stomatal closure through SA- and ABA- } \\
\text { mediated signaling }\end{array}$ & $\begin{array}{l}\text { Chr5: M- } \\
\text { QTL4 }\end{array}$ & Seedling \\
\hline $\begin{array}{l}\text { LOC_- } \\
\text { Os05g39770.1 }\end{array}$ & Aminotransferase, putative, expressed & $\begin{array}{l}\text { Chr5:M- } \\
\text { QTL4 }\end{array}$ & Leaves \\
\hline $\begin{array}{l}\text { LOC_- } \\
\text { Os05g38660.1 }\end{array}$ & Expressed protein & $\begin{array}{l}\text { Chr5:M- } \\
\text { QTL4 }\end{array}$ & Seedling \\
\hline $\begin{array}{l}\text { LOC_- } \\
\text { Os05g40010.1 }\end{array}$ & LTPL17, Protease inhibitor/seed storage/LTP family protein precursor, Signal domain & $\begin{array}{l}\text { Chr5:M- } \\
\text { QTL4 }\end{array}$ & Seedling \\
\hline $\begin{array}{l}\text { LOC_- } \\
\text { Os05g41670.1 }\end{array}$ & Expressed protein & $\begin{array}{l}\text { Chr5:M- } \\
\text { QTL4 }\end{array}$ & Seedling \\
\hline $\begin{array}{l}\text { LOC_ } \\
\text { Os05g39990.1 }\end{array}$ & Plant-type cell wall organization & $\begin{array}{l}\text { Chr5:M- } \\
\text { QTL4 }\end{array}$ & Root \\
\hline $\begin{array}{l}\text { LOC_- } \\
\text { Os05g39250.1 }\end{array}$ & Phosphatidylethanolamine & $\begin{array}{l}\text { Chr5:M- } \\
\text { QTL4 }\end{array}$ & Root \\
\hline $\begin{array}{l}\text { LOC_- } \\
\text { Os06g48860.1 }\end{array}$ & OsSAUR28, Auxin-responsive SAUR gene family member, expressed & $\begin{array}{l}\text { Chr6:M- } \\
\text { QTL4 }\end{array}$ & Root \\
\hline $\begin{array}{l}\text { LOC__ } \\
\text { Os06g } 48810.1\end{array}$ & OsHKTI, $\mathrm{Na}^{+}$transporter, $\mathrm{k}^{+}$transporter,cation transmembrane transporter activity & $\begin{array}{l}\text { Chr6: M- } \\
\text { QTL4 }\end{array}$ & Root and Leaves \\
\hline $\begin{array}{l}\text { LOC_ } \\
\text { Os06g48300.1 }\end{array}$ & $P P 2 C$, protein serine/threonine phosphatase activity & $\begin{array}{l}\text { Chr6: M- } \\
\text { QTL4 }\end{array}$ & $\begin{array}{l}\text { Root, Seedling \& } \\
\text { leaves }\end{array}$ \\
\hline $\begin{array}{l}\text { LOC_ }_{-} \\
\text {Os06g49190.1 }\end{array}$ & LTPL154, Protease inhibitor/seed storage/LTP family protein precursor, Signal domain & $\begin{array}{l}\text { Chr6:M- } \\
\text { QTL4 }\end{array}$ & Seedling \\
\hline
\end{tabular}

QTL1 were up-regulated in the seedlings under salinity stress (Table S6, Fig. 6). Furthermore, OsCIPK24 (SOS2) in Chr6:M-QTL3 and OsCIPK10 in Chr3:M-QTL2 were up-regulated in the seedlings (Table S6, Fig. 4). CIPK (CBL- Interacting Protein Kinases) pathway has emerged as a main signaling pathway and adjusts the salt tolerance in rice $[42,43]$. A generic signal transduction pathway starts with signal perception, followed by the generation of the second messengers)e.g., inositol phosphates and Reactive Oxygen Species (ROS)) and the transcription factors controlling the specific sets of stress-regulated genes [44].

\section{Transcription regulation}

Transcription factors are important for emergence of any phenotype, as they are able to regulate the expression of all the related genes [32]. HSFA6B (located in Chr1:M-QTL3, up-regulated in the seedlings) acts as a 


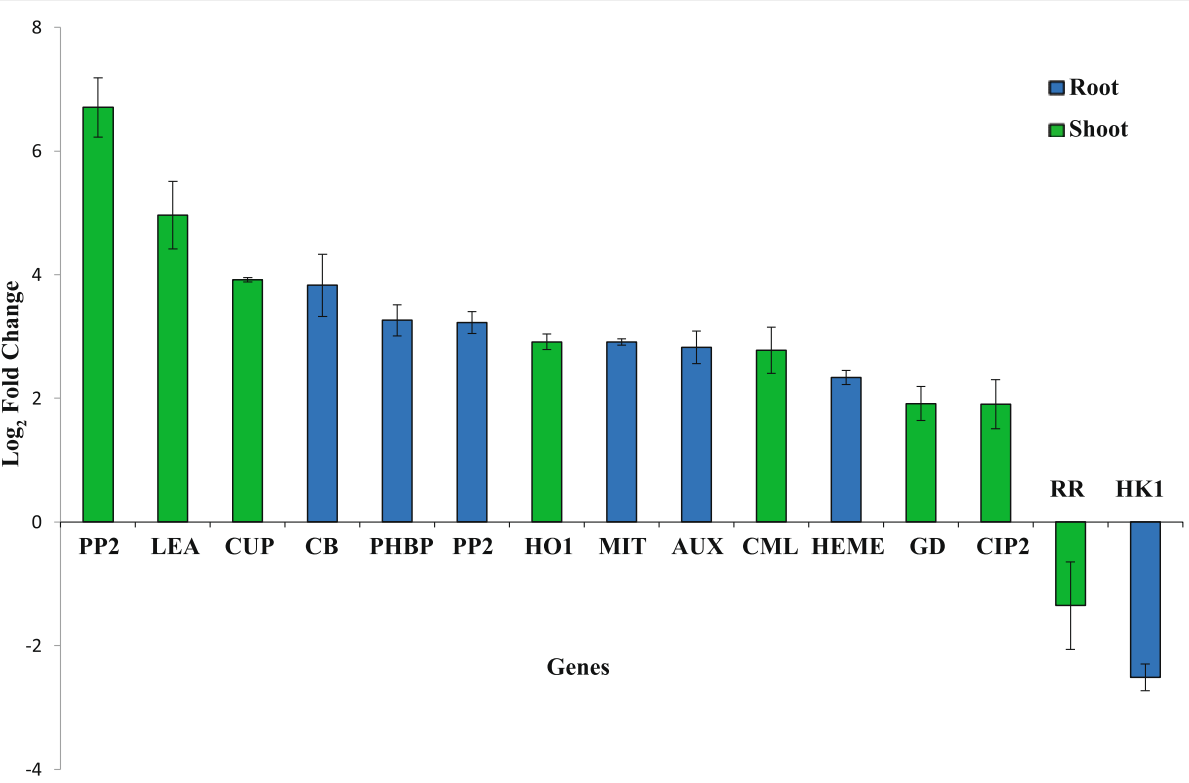

Fig. 5 Validation of selected genes using qRT-PCR in root and shoot tissues of FL478 (tolerant genotype). Bar graphs depict the relative transcript abundance of the selected potential candidate genes in FL478 under different conditions. Data points are represented as log2 fold change values

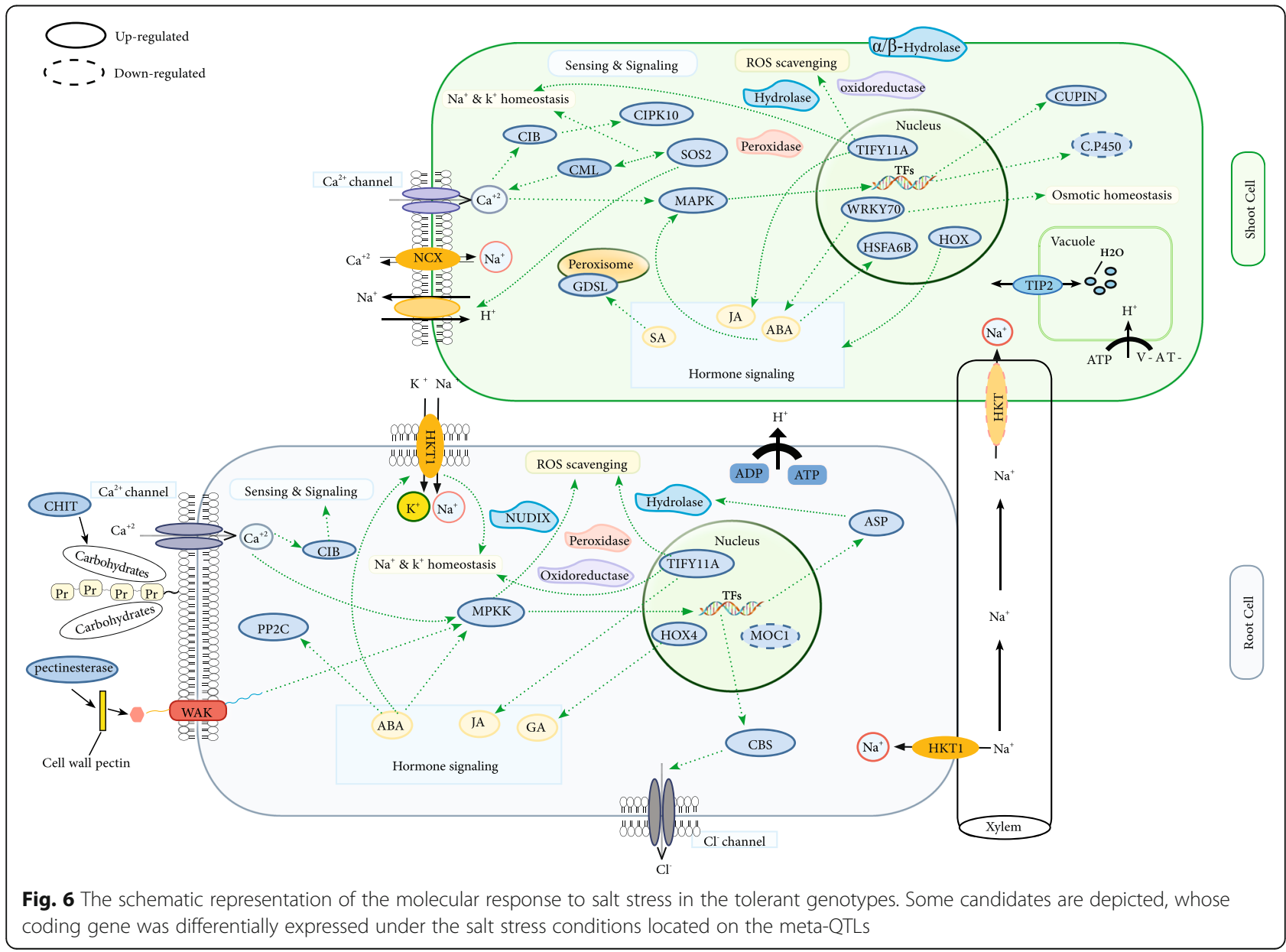


positive regulator downstream of Abscisic Acid (ABA) signaling directly bound to the promoter of Dehydration-Responsive Element-Binding (DREB) and increasing its expression (Table S6, Fig. 6). Upregulation of the Dehydration-Responsive Element -Binding protein 2A (DREB2A) can activate the various genes related to stress tolerance in different plant species [45]. It has also been reported that over-expression of OsTIFY11 (located in Chr3:M-QTL1, up-regulated in the shoot and root) increased the tolerance to salinity stress through the Jasmonic Acid (JA) signaling and through modulating the potassium homeostasis (Table S6, Fig. 6) [46]. There were OsHOX22 and OsHOX24 from homeobox familyin Chr3:M-QTL1 and Chr4:M-QTL3, respectively, which were both up-regulated in the seedlings (Table S6, Fig. 6). OsHOX24 was the most up-regulated gene under $150 \mathrm{mM} \mathrm{NaCl}$ in the salt tolerant genotype (FL478); while it was highly down-regulated in the salt sensitive genotype (IR29) [31]. Also, the role of OsHOX24 has been already found to regulate the abiotic stress responses through fine tuning the expression of stressresponsive genes in rice [47]. Moreover, there was OsWRKY70 in Chr5:M-QTL4 and up-regulated in the seedlings (Table S6, Fig. 6). It has been stated that OsWRKY70 as a negative regulator of stomatal closure through SA- and ABA-mediated signaling, play important role in the plant tolerance to osmotic stress [48]. Moreover, GRAS (located in Chr5:M-QTL4 and downregulated in the roots) proteins belong to a plantspecific transcription factor family involved in many plant processes including plant growth and development as well as abiotic stress responses (Table S6, Fig. 6) [49, 50]. It has also been reported that MOC1 encodes a nuclear transcription factor from GRAS family. MOC1 acts as a positive regulator of lateral branching or increased tiller number [51].

\section{ROS inhibition}

One of the key mechanisms to increase the plants adaptation to detrimental environmental conditions including high salt concentrations is regulation of the toxic ROS levels [33, 52]. Nudix hydrolase was found in Chr4: M-QTL3 and was up-regulated in the roots (Table S6, Fig. 6), generally removing the excess toxic metabolites or controlling the accessibility of intermediates in the metabolic pathways [53]. Also, there was a peroxidase coding gene belongs to the antioxidant system in Chr1:M-QTL2 that was up-regulated in the leaves (Table S6, Fig. 6). Transgenic Arabidopsis plants expressing the cytosolic peroxidase genes have been reported to show higher salt tolerance [20]. In addition, there was a hydrolase coding gene belonging to the alpha/beta fold family domain containing protein in Chr3:M-QTL3that was up-regulated in the seedling (Table S6, Fig. 6). It has been reported that overexpression of a gene coding $\alpha / \beta$-hydrolase fold enzyme led to significantly higher salinity tolerance compared to the wild-type because of protecting the membrane integrity and increasing the ROS scavenging capacity in the sweetpotato [54].

\section{Ionic homeostasis}

Regulation of the ion flux under salinity stress is necessary for the cells to keep the concentrations of toxic ions at low levels and to collect the essential ions. Salinity stress up-regulates the trasporter encoding genes such as $\mathrm{Na}^{+}$and $\mathrm{K}^{+}$transporters and vacuolar $\mathrm{Na}^{+} / \mathrm{H}^{+}$exchangers [55]. Several transporters were observed in the meta-QTL positions among which, HKT1 was found in Chr6: M-QTL4; down-regulated in the leaves and upregulated in the roots (hotspot-region, Table S6, Fig. 6). High affinity $\mathrm{K}^{+}$transporter known as $\mathrm{Na}^{+} / \mathrm{K}^{+}$cotransporters reduces the transport of $\mathrm{Na}^{+}$to the shoots and positively regulate the salinity tolerance in rice and Arabidopsis [56]. Two genes encoding the vacuolar protein with signal peptide domain were identified in Chr1: M-QTL3 and Chr3:M-QTL2 (Table S6); up-regulated in the seedling. The genes coding the sodium/calcium exchanger (NCX) in Chr12:M-QTL4; up-regulated in seedling (Table S6, Fig. 6), which play significant roles in $\mathrm{Ca}^{2+}$ signaling and ion homeostasis. Sodium/calcium exchangers use the $\mathrm{Na}^{+}$electrochemical gradient through the plasma membrane to extrude the intracellular $\mathrm{Ca}^{2+}[57,58]$.

\section{Other salt tolerance related potential candidate genes}

Twenty three unknown potential candidate genes were found among which, five genes possess the CBS or cupin domain(s) in their sequence. For instance, a gene containing CBS domain was located in Chr2:M-QTL1 that up-regulated in the roots. Previous reports have indicated that, it plays a role in the salinity and oxidative stress tolerance through influencing the chloride channels (Kushwaha et al. 2009). It has also been reported that overexpression of OsCBSX4 improved the tolerance against salinity and oxidative stress in tobacco transgenic lines [59].

Furthermore,four genes possessing the cupin domain(s) in their sequence were found in various M-QTL positions (Table S6) while there were up-regulated in the seedlings. According to the previous reports, cupin domain might play a role in improving the seed germination in rice under salinity stress because the proteins having the cupin domain(s) were observed near the position of QTLs related to the seed dormancy, seed reserve utilization, and seed germination [60]. 


\section{Conclusions}

To inspect the molecular mechanisms by which tolerant genotypes respond to the salinity stress, we employed an integrative approach to identify candidate genes related to salt tolerance in rice. The obtained results indicated that, the salt tolerant genotypes utilize more effective mechanisms in response to the salt stress (Fig. 6) particularly in terms of 1) Sensing and signalling of the salt stress; several genes coding the cell wall organization, pectinesterase, Ser/Thr phosphatase, chitinase, CIPKwere observed in the hotspot-regions that were differentially expressed in the tolerant genotypes. 2) Regulation of transcription; several salinity responsive transcription factors (TFs) belonging to different families including TIFY, MYB, HSF, HOX, WRKY, AP2, and GRAS families were found both in the meta-QTL regions and among the DEGs, which have been shown to play essential roles in the salinity tolerance in rice. 3) Ionic and osmotic homeostasis; some transporters were also among the promising candidate genes such as $H K T 1(\mathrm{Na} / \mathrm{K}$ transporter), NCX (sodium/calcium exchanger), and TIP2-1 (aquaporin). 4) ROS scavenging; there were many important genes involved in detoxification such as hydrolase, oxidoreductase, and peroxidase among the DEGs that were located in the meta-QTL positions. Further research on these promising candidate genes can bring about beneficial information which would be used to improve salt tolerance in the given genotypes through genetic engineering or molecular breeding.

\section{Methods}

\section{Meta-analysis of QTLs}

\section{Preparing the QTL data}

All the reported QTLs related to the salinity tolerance in rice (from 2009 to 2018) were collected including those identified in 15 previously published studies [9-14, 16, 61-68]. The QTLs data including the parental lines, the type and size of QTL mapping population, and the number of QTLs per trait were provided. Moreover, the flanking molecular markers, Confidence Interval (CI), QTL position, Logarithm of the Odds (LOD) score, and Proportion of Phenotypic Pariance Explained (PVE or R2) were evaluated with respect to each QTL. The QTLs used in this study were derived from various population types (including: F2, backcrossed lines (BC3F4), Recombinant Inbred Lines (RILs)), and sizes (from 87 to 285 plants) from different tissues at seedling and reproductive developmental stages (Table S1).

\section{Consensus map and QTL projection}

The consensus QTL regions were identified using the BioMercator software [69]. The map of the International Rice Microsatellite Initiative (IRMI) available at https:// archive.gramene.org (IRMI_2003) was used as the reference map for Meta-QTL analysis. The 95\% CI of the initial QTL was computed using the following formulas before projecting the QTLs on the consensus map:

(i) For F2 lines: $C I=\frac{530}{N \times R^{2}}$

(ii) For Double Haploid (DH) lines: $C I=\frac{287}{N \times R^{2}}$

(iii) For RILs: $C I=\frac{163}{N \times R^{2}}$

Where, $\mathrm{N}$ is the population size and $R^{2}$ is the percentage of phenotypic variation explained by the related QTL. The scaling rule between the marker intervals of the initial QTLs was used for the QTL positions on the consensus chromosome map.

\section{Meta-analysis of the QTLs}

Meta analysis was performed by the default parameter sets in the BioMercator V4.2 tool. The consensus QTL was calculated as 1, 2, 3, and n models by the software. The Akaike Information Criterion (AIC) was used to select the QTL models on each chromosome [70]. According to the AIC value, the QTL model with the lowest AIC value was considered as a significant model.

\section{RNA -sequencing}

RNA-Seq data was obtained from our previous study on two contrasting genotypes of Oryza sativa under salinity stress [31]. Briefly, the young seedlings of FL478 (Salt tolerant) and IR29 (Salt sensitive) were treated with 150 $\mathrm{mM} \mathrm{NaCl}$ and the root samples were collected $24 \mathrm{~h}$ after inception of the salt stress. Along with, normal samples (at the same conditions but without salinity treatment) were also collected as control samples [31]. The purified RNA was used to construct the cDNA library; the qualified libraries were subsequently sequenced using IlluminaHiSeq $^{\text {Tx }} 2500$ sequencer. The transcriptome raw data including control (SRR7944745 and SRR7944784) and salt treated samples (SRR7944792 and SRR7944793) of FL478, and control samples (SRR7945188 and SRR7945229) and salt treated samples (SRR7945230 and SRR7945234) of IR29 are available at SRA (Sequence Read Achieve) of NCBI database. The quality of datasets was conducted using the FastQC tool [71]. TopHat was used to map eight paired-end sequencing libraries of two rice genotypes against the rice reference genome sequences IRGSP 1.0 (ftp://ftp.ensemblgenomes.org/pub /plants) [71]. Raw sequencing reads were then assembled through Cufflinks and Cuffmerge meta assembler utilities [71]. Finally, DEGs were identified by Cuffdiff utility, with $\log 2$ fold change $\geq 1$ (up-regulated genes) and $\leq(-1)$ (down-regulated genes) and $Q$-value cut-off of $\leq 0.05$. 


\section{Meta-analysis of the gene expression data according to tissues \\ RNA-seq Meta-dataset}

The available (by the time of this analysis) transcriptome datasets of rice plants exposed to salinity stress were collected from the National Center for Biotechnology (NCBI) database (Table S7). The genes with $-1 \geq \log 2$ fold change $\geq 1$ and significant Q-value (FDR $\leq 5 \%$ ) were considered as DEGs from these RNA-seq datasets and were classified into four tissues (i.e. shoot, root, seedling, and leaves).

\section{Microarray Meta-analysis}

Rice expression data subjected to salt stress were obtained from the NCBI's Gene Expression Omnibus repository (GEO) [71, 72]. Totally, nine GEO datasets were downloaded from the affymetrix platform Rice Genome Array (Affymetrix or Agilent microarray platforms) (Table S5). Each set of the expression data was preprocessed separately. The LIMMA package in the $\mathrm{R}$ program was used to analyze Agilent microarray data [73], while affymetrix platforms were handled in the $R$ program by the Affy package. The raw data of each source was preprocessed by the quantile normalization and Robust Multi-Array Average background correction. Then, the probes with low-intensity and noninformative were removed from the program standard settings; then, the probes were transformed to their related genomic location. The RMA was employed for normalization of values for the subsequent MA. Then, the difference between each treatment and its control was computed using the LIMMA package. After fitting the data into a linear model, simple empirical Bayes model was used to revise the standard errors. For each contrast in every gene, moderated t-statistic and logodds of differential expression were calculated. The genes with $-1 \geq \log 2$ fold change $\geq 1$ and $Q$-value cutoff of $\leq 0.05$ were determined as DEGs in each of the four tissues.

\section{Integration of significant gene expressions and literature citations for the DEGs}

A novel data processing pipeline was proposed in this research integrating different data types to identify promising candidate genes related to salt tolerance in rice (Fig. 4). On one hand, DEGs were integrated in response to salinity stress in rice from both microarray and RNA-Seq technologies. On the other hand, NCBI (NCBI; www.ncbi.nlm.nih.gov) literature was searched to identify the published reports on the salinity tolerance genes in rice. In this research, 111 papers were reviewed and all the reported salinity tolerance-related genes in rice were collected. All the identified genes were classified into four tissues (including shoot, root, seedling, and leaves) (Table S8). Venn diagram (using the R package) was used to compare the overlaps in the detected genes for each tissue using different approaches (including RNA-seq, microarray, and literature review) and the common genes were detected. Finally, salinity tolerance associated meta-QTLs regions were explored to find the DEGs, which are coincided with the meta-QTL positions. For identification of DEGs in meta-QTLs regions, the flanking markers of the identified MQTLs were used to detect the physical intervals for each meta-QTL. Then, the genes located in meta-QTLs regions were found according to the rice genome assembly IRGSP 1.0.

\section{Functional annotation and pathway analysis}

Enrichment analysis of the DEGs were performed using the AgriGO public web tool [74]. The over-represented GO terms were filtered in the three main categories including the "Biological Process", "Molecular Function" and "Cellular Component" using the Fisher's exact test (Q-value < 0.05) and were corrected by the False Discovery Rate (FDR) method at $p<0.05$.

\section{Identification of salinity tolerance-related candidate genes in the meta-QTL regions}

The genes observed at least by two approaches (from the three applied methodologies including RNA-seq, microarray and literature review) were called as common genes in this paper.The common genes were sought in the salinity tolerance associated meta-QTLs regions to find the potential candidate genes. The potential candidate genes located on the hotspot-regions overlying original QTLs for both yield components and ion homeostasis traits were assumed as promising candidate genes (Fig. 4).

\section{Plant growth and salt stress treatment}

Seeds of FL478 as the salt tolerant rice (Oryza sativa L.) genotype were provided from International Rice Research Institute (IRRI). Seeds sterilization and germination, as well as plant growth conditions were performed as previously described [31]. Root and shoot samples of 21-days-old treated seedlings with $150 \mathrm{mM} \mathrm{NaCl}$ were collected $24 \mathrm{~h}$ after inception of the salt stress, instantly put in liquid nitrogen and kept at $-80^{\circ} \mathrm{C}$ until RNA extraction.

\section{RNA extraction and CDNA library synthesis}

Total RNA extraction was performed by the RNeasy Plant kit (Qiagen) from $100 \mathrm{mg}$ of shoot and root tissues. Integrity and quality of RNA samples was inspected using a NanoDrop ND-1000 ${ }^{\circ}$ spectrophotometer and agarose gel electrophoresis. The cDNA library synthesis was done using iScriptTM cDNA synthesis kit (BioBasic) consistent with the manufacturer's instructions. 


\section{Validation of salinity tolerance-related candidate genes by qRT-PCR assay}

A total of 15 genes from the list of possible candidate genes were randomly nominated in each tissue (Table S6) for validation by quantitative real-time PCR (RTqPCR). Specific primer pairs for each gene (Table S9 for list primer) were designed by Oligo 7.0 (National Bioscience Inc., Plymouth, USA). The qRT-PCR with three independent biological replicates was done by a LightCycler 96 Real-Time PCR System (Roche Life Science, Germany) and SYBR Premix without ROX based on manufacturer's protocol. Actin gene of rice (OS03G0836000) was employed as a suitable inner control gene. Transcript levels of nominated genes from three biological replicates were computed as 2- $\Delta \Delta \mathrm{Ct}$ [75].

\section{Supplementary information}

Supplementary information accompanies this paper at https://doi.org/10. 1186/s12870-020-02679-8.

Additional files 1: Table S1. List of the QTL mapping studies used for meta-QTL analysis for traits associated with the salt tolerance in rice. Table S2. The summary of the original QTLs related to salt tolerance traits. Table S3. The Summary of the original QTLs related to salinity tolerance included in the meta-analysis. Table S4. The consensus QTLs of 32 traits identified by meta-analysis in rice. Table S5. The original microarray datasets selected for meta-analysis of rice under salinity stress.

Table S6. The list of possible candidate genes in the meta-QTL regions (The asterisk on meta position column, represents promising genes located in the hotspot positions). Table S7. The list of publicly accessible RNA-seq datasets was used in this study. Table S8. The list of reported salinity tolerance related genes in rice based on the literature review, classified into four tissues (including shoot, root, seedling, and leaves) in 4 sheets. Table $\mathbf{S} 9$. List of primers used for qRT-PCR analysis. Fig. S1. Number of the original QTLs that are associated with each salt tolerance related trait (Traits along with their abbreviations are provided in Table S2). Fig. S2. Number of the original QTLs related to the salt tolerance in each chromosome of rice. Fig. S3. Number of differentially expressed genes (DEGs) identified by RNA-seq meta-analysis in four tissues (including shoot, root, seedling, and leaves). Fig. S4. Number of differentially expressed genes (DEGs) identified by microarray meta-analysis in four tissues (including shoot, root, seedling and leaves). Fig. S5. GO term assignment of the identified DEGs located in the meta-QTL positions to three main categories of cellular component, molecular function, and biological process. Fig. S6. Graph illustrating of the melt curves from qRTPCR of the selected potential candidate genes in FL478.

\section{Abbreviations \\ DEGs: Differentially expressed genes; QTL: Quantitative trait locus; MA: Meta- analysis; ROS: Reactive oxygen species; KLV: $\mathrm{K}^{+}$in leaf vegetative; NS: Shoot sodium concentration; NKS: Ratio of the shoot sodium and potassium concentration; KS: Shoot potassium concentration; RN: Root $\mathrm{Na}^{+}$concentration; SIS: Salt injury score; QGW: 1000-grain weight (g); DF: Days to flowering; NFS: Number of fertile spikelets; BP: Biological Processes; GO: Gene Ontology; MF: Molecular Function; CC: Cellular Component; M-QTL: Meta-QTL; $\mathrm{Cl}$ : Confidence Interval; LOD: Logarithm of the odds ratio; AIC: Akaike Information Criterion}

\section{Acknowledgments}

The authors are grateful to Agricultural Biotechnology Research Institute of Iran (ABRII) for the supports, and Mr. Mohammad Jedari to help in creating the artworks.

\section{Authors' contributions}

RMM conducted the experiments, and drafted the manuscript. Z-SS conceived the project, supervised and coordinated the research, also revised the manuscript. RMM analyzed RNA- seq data, RMM and PD performed the meta-QTL analysis, RMM and S-MM analyzed Microarray data. NBJ and MRG checked the final manuscript.

\section{Funding}

This study was financially supported by Biotechnology Development Council of the Islamic Republic of Iran)grant No: 950618(and Iran National Science Foundation (INSF grant No: 98014939).

\section{Availability of data and materials}

Accession codes: All primary sequence read data has been deposited in NCBI database under BioProject ID: PRJNA493951 and PRJNA493923. All data supporting the conclusions of this article are provided within the article and its supplementary (Additional file 1: Table S1, Table S2, Table S3, Table S4, Table S5, Table S6, Table S7, Table S8, Table S9, Fig. S1, Fig. S2, Fig. S3, Fig. S4, Fig. S5, Fig. S6).

Ethics approval and consent to participate Not applicable.

\section{Consent for publication}

Not applicable.

\section{Competing interests}

The authors declare that they have no competing interests.

\section{Author details}

'Department of Systems Biology, Agricultural Biotechnology Research Institute of Iran (ABRII), Agricultural Research, Education and Extension Organization (AREEO), PO Box 31535-1897, Karaj, Iran. ${ }^{2}$ Faculty of Crop Science, Department of Plant breeding and Biotechnology, Sari Agricultural Science and Natural Resources University, Sari, Iran.

Received: 5 October 2019 Accepted: 24 September 2020

Published online: 01 October 2020

\section{References}

1. ZHANG H, et al. Progress of potato staple food research and industry development in China. J Integr Agric. 2017;16(12):2924-32.

2. Xing Y, Zhang Q. Genetic and molecular bases of rice yield. Annu Rev Plant Biol. 2010;61:421-42

3. Hoang $T$, et al. Improvement of salinity stress tolerance in rice: challenges and opportunities. Agronomy. 2016;6(4):54.

4. Frouin J, et al. Tolerance to mild salinity stress in japonica rice: a genomewide association mapping study highlights calcium signaling and metabolism genes. PLoS One. 2018;13(1):e0190964.

5. Zeng L, Shannon MC, Lesch SM. Timing of salinity stress affects rice growth and yield components. Agric Water Manag. 2001;48(3):191-206.

6. Zhao $X$, et al. Comparative metabolite profiling of two rice genotypes with contrasting salt stress tolerance at the seedling stage. PLoS One. 2014;9(9): e108020.

7. Ray DK, et al. Climate variation explains a third of global crop yield variability. Nat Commun. 2015;6:5989.

8. Joseph B, Jini D, Sujatha S. Biological and physiological perspectives of specificity in abiotic salt stress response from various rice plants. Asian J Agric Sci. 2010;2(3):99-105.

9. Puram VRR, Ontoy J, Subudhi PK. Identification of QTLs for salt tolerance traits and prebreeding lines with enhanced salt tolerance in an introgression line population of rice. Plant Mol Biol Report. 2018:1-15.

10. Mohammadi R, et al. Mapping quantitative trait loci associated with yield and yield components under reproductive stage salinity stress in rice (Oryza sativa L.). J Genet. 2013;92(3):433-43.

11. Wang $\mathrm{S}$, et al. Integrated RNA sequencing and QTL mapping to identify candidate genes from Oryza rufipogon associated with salt tolerance at the seedling stage. Front Plant Sci. 2017;8:1427.

12. De Leon TB, Linscombe S, Subudhi PK. Identification and validation of QTLS for seedling salinity tolerance in introgression lines of a salt tolerant rice landrace 'Pokkali'. PLoS One. 2017;12(4):e0175361. 
13. Kim D-M, et al. Mapping QTLs for salt tolerance in an introgression line population between japonica cultivars in rice. J Crop Sci Biotechnol. 2009; 12(3):121

14. Liang J -l, et al. Identification of QTLS associated with salt or alkaline tolerance at the seedling stage in rice under salt or alkaline stress. Euphytica. 2015;201(3):441-52.

15. Pandit A, et al. Combining QTL mapping and transcriptome profiling of bulked RILs for identification of functional polymorphism for salt tolerance genes in rice (Oryza sativa L.). Mol Gen Genomics. 2010;284(2):121-36.

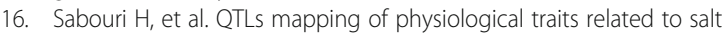
tolerance in young rice seedlings. Biol Plant. 2009;53(4):657-62.

17. Rahman MA, et al. Exploring novel genetic sources of salinity tolerance in rice through molecular and physiological characterization. Ann Bot. 2016; 117(6):1083-97.

18. Ren $\mathrm{Z}-\mathrm{H}$, et al. A rice quantitative trait locus for salt tolerance encodes a sodium transporter. Nat Genet. 2005;37(10):1141.

19. Barnes $M$, et al. Experimental comparison and cross-validation of the Affymetrix and Illumina gene expression analysis platforms. Nucleic Acids Res. 2005;33(18):5914-23.

20. Lu Z, Liu D, Liu S. Two rice cytosolic ascorbate peroxidases differentially improve salt tolerance in transgenic Arabidopsis. Plant Cell Rep. 2007;26(10):1909-17.

21. Garber $M$, et al. Computational methods for transcriptome annotation and quantification using RNA-seq. Nat Methods. 2011;8(6):469.

22. Xu H-M, et al. Transcriptome analysis of Brassica napus pod using RNA-Seq and identification of lipid-related candidate genes. BMC Genomics. 2015; 16(1):858

23. Ramasamy A, et al. Key issues in conducting a meta-analysis of gene expression microarray datasets. PLoS Med. 2008;5(9):e184.

24. Tseng GC, Ghosh D, Feingold E. Comprehensive literature review and statistical considerations for microarray meta-analysis. Nucleic Acids Res. 2012;40(9):3785-99.

25. Price AH. Believe it or not, QTLs are accurate! Trends Plant Sci. 2006;11(5): 213-6.

26. Islam M, Ontoy J, Subudhi PK. Meta-Analysis of Quantitative Trait Loci Associated with Seedling-Stage Salt Tolerance in Rice (Oryza sativa L.). Plants. 2019;8(2):33.

27. Swamy BM, Sarla N. Meta-analysis of yield QTLs derived from inter-specific crosses of rice reveals consensus regions and candidate genes. Plant Mol Biol Report. 2011;29(3):663-80.

28. Wu Y, et al. Quantitative trait loci identification and meta-analysis for rice panicle-related traits. Mol Gen Genomics. 2016;291(5):1927-40.

29. Courtois $B$, et al. Rice root genetic architecture: meta-analysis from a drought QTL database. Rice. 2009;2(2):115.

30. Zhang $H$, et al. Meta-analysis and candidate gene mining of lowphosphorus tolerance in maize. J Integr Plant Biol. 2014;56(3):262-70.

31. Mirdar Mansuri R, et al. Dissecting molecular mechanisms underlying salt tolerance in rice: a comparative transcriptional profiling of the contrasting genotypes. Rice. 2019;12(1):13.

32. Ganie SA, et al. Advances in understanding salt tolerance in rice. Theor Appl Genet. 2019:1-20.

33. Landi S, et al. Poaceae vs. abiotic stress: focus on drought and salt stress, recent insights and perspectives. Front Plant Sci. 2017;8:1214.

34. Zagorchev L, Kamenova P, Odjakova M. The role of plant cell wall proteins in response to salt stress. Sci World J. 2014;2014.

35. Decreux A, Messiaen J. Wall-associated kinase WAK1 interacts with cell wal pectins in a calcium-induced conformation. Plant Cell Physiol. 2005;46(2): 268-78.

36. País SM, et al. Characterization of potato (Solanum tuberosum) and tomato (Solanum lycopersicum) protein phosphatases type 2A catalytic subunits and their involvement in stress responses. Planta. 2009;230(1):13-25.

37. País SM, Téllez-Iñón MT, Capiati DA. Serine/threonine protein phosphatases type 2A and their roles in stress signaling. Plant Signal Behav. 2009;4(11): 1013-5.

38. Yu RMK, et al. Structure, evolution and expression of a second subfamily of protein phosphatase $2 \mathrm{~A}$ catalytic subunit genes in the rice plant (Oryza sativa L.). Planta. 2005;222(5):757-68.

39. Yu RMK, et al. Two genes encoding protein phosphatase $2 \mathrm{~A}$ catalytic subunits are differentially expressed in rice. Plant Mol Biol. 2003;51(3):295-311.

40. Xu C, et al. A wheat (Triticum aestivum) protein phosphatase 2A catalytic subunit gene provides enhanced drought tolerance in tobacco. Ann Bot. 2007;99(3):439-50.
41. Mishra NS, Tuteja R, Tuteja N. Signaling through MAP kinase networks in plants. Arch Biochem Biophys. 2006:452(1):55-68.

42. Liu W-Z, et al. Rapeseed calcineurin B-like protein CBL4, interacting with CBL-interacting protein kinase CIPK24, modulates salt tolerance in plants. Biochem Biophys Res Commun. 2015;467(3):467-71.

43. Abdula SE, et al. Overexpression of BrCIPK1 gene enhances abiotic stress tolerance by increasing proline biosynthesis in rice. Plant Mol Biol Report. 2016;34(2):501-11.

44. Xiong L, Schumaker KS, Zhu J-K. Cell signaling during cold, drought, and salt stress. Plant Cell. 2002;14(suppl 1):S165-83.

45. Huang $B$, et al. Cloning and characterization of the dehydration-responsive element-binding protein 2A gene in Eruca vesicaria subsp sativa. Genet Mol Res. 2016:15:23-9.

46. Ye $\mathrm{H}$, et al. Identification and expression profiling analysis of TIFY family genes involved in stress and phytohormone responses in rice. Plant Mol Biol. 2009;71(3):291-305.

47. Bhattacharjee A, Sharma R, Jain M. Over-expression of OsHOX24 confers enhanced susceptibility to abiotic stresses in transgenic rice via modulating stress-responsive gene expression. Front Plant Sci. 2017:8:628.

48. Li J, et al. Defense-related transcription factors WRKY70 and WRKY54 modulate osmotic stress tolerance by regulating stomatal aperture in Arabidopsis. New Phytol. 2013;200(2):457-72.

49. Grimplet J, et al. Structural and functional analysis of the GRAS gene family in grapevine indicates a role of GRAS proteins in the control of development and stress responses. Front Plant Sci. 2016;7:353.

50. Li P, et al. BrLAS, a GRAS transcription factor from Brassica rapa, Is Involved in Drought Stress Tolerance in Transgenic Arabidopsis. Front Plant Sci. 2018, 9:1792.

51. Sakamoto T, Matsuoka M. Identifying and exploiting grain yield genes in rice. Curr Opin Plant Biol. 2008;11(2):209-14.

52. Hossain MS, Dietz K-J. Tuning of redox regulatory mechanisms, reactive oxygen species and redox homeostasis under salinity stress. Front Plant Sci. 2016;7:548.

53. Corpas FJ, et al. Activation of NADPH-recycling systems in leaves and roots of Arabidopsis thaliana under arsenic-induced stress conditions is accelerated by knock-out of Nudix hydrolase 19 (AtNUDX19) gene. J Plant Physiol. 2016;192:81-9.

54. Liu $D$, et al. A novel $a / \beta$-hydrolase gene IbMas enhances salt tolerance in transgenic sweetpotato. PLoS One. 2014;9(12):e115128.

55. Zhu J-K. Regulation of ion homeostasis under salt stress. Curr Opin Plant Biol. 2003;6(5):441-5

56. Wang $\mathrm{R}$, et al. The rice high-affinity potassium transporter $1 ; 1$ is involved in salt tolerance and regulated by an MYB-type transcription factor. Plant Physiol. 2015;168(3):1076-90.

57. Liao J, et al. Mechanism of extracellular ion exchange and binding-site occlusion in a sodium/calcium exchanger. Nat Struct Mol Biol. 2016;23(6):590.

58. Giladi M, Tal I, Khananshvili D. Structural features of ion transport and allosteric regulation in sodium-calcium exchanger (NCX) proteins. Front Physiol. 2016:7:30.

59. Singh AK, et al. Overexpression of rice CBS domain containing protein improves salinity, oxidative, and heavy metal tolerance in transgenic tobacco. Mol Biotechnol. 2012;52(3):205-16.

60. $\mathrm{Xu} \mathrm{E}$, et al. Proteomic analysis reveals proteins involved in seed imbibition under salt stress in rice. Front Plant Sci. 2017;7:2006.

61. Puram VRR, et al. Genetic dissection of seedling stage salinity tolerance in rice using introgression lines of a salt tolerant landrace Nona Bokra. J Hered. 2017;108(6):658-70

62. Wang Z, et al. QTL analysis of $\mathrm{Na}+$ and $\mathrm{K}+$ concentrations in roots and shoots under different levels of $\mathrm{NaCl}$ stress in rice (Oryza sativa L.). PLoS One. 2012;7(12):e51202.

63. Pandit A, et al. Combining QTL mapping and transcriptome profiling of bulked RILs for identification of functional polymorphism for salt tolerance genes in rice (Oryzasativa L.). Mol Gen Genomics. 2010;284(2):121-36.

64. Thomson MJ, et al. Characterizing the Saltol quantitative trait locus for salinity tolerance in rice. Rice. 2010;3(2):148.

65. Tian $L$, et al. Identification of quantitative trait loci associated with salt tolerance at seedling stage from Oryza rufipogon. J Genet Genomics. 2011; 38(12):593-601.

66. Wang Z, et al. Identification of QTLs with main, epistatic and QTLX environment interaction effects for salt tolerance in rice seedlings under different salinity conditions. Theor Appl Genet. 2012;125(4):807-15. 
67. Zheng $\mathrm{H}$, et al. QTL analysis of $\mathrm{Na}+$ and $\mathrm{K}+$ concentrations in shoots and roots under $\mathrm{NaCl}$ stress based on linkage and association analysis in japonica rice. Euphytica. 2015;201(1):109-21.

68. Cheng $L$, et al. Identification of salt-tolerant QTLs with strong genetic background effect using two sets of reciprocal introgression lines in rice. Genome. 2011:55(1):45-55.

69. Sosnowski O, Charcosset A, Joets J. BioMercator V3: an upgrade of genetic map compilation and quantitative trait loci meta-analysis algorithms. Bioinformatics. 2012;28(15):2082-3.

70. Goffinet B, Gerber S. Quantitative trait loci: a meta-analysis. Genetics. 2000; 155(1):463-73.

71. Trapnell C, et al. Differential gene and transcript expression analysis of RNAseq experiments with TopHat and cufflinks. Nat Protoc. 2012;7(3):562.

72. Barrett T, et al. NCBI GEO: archive for functional genomics data sets—update. Nucleic Acids Res. 2012:41(D1):D991-5

73. Smyth GK. Limma: linear models for microarray data, in Bioinformatics and computational biology solutions using R and Bioconductor: Springer; 2005. p. 397-420.

74. Tian T, et al. agriGO v2. 0: a GO analysis toolkit for the agricultural community, 2017 update. Nucleic Acids Res. 2017;45(W1):W122-9.

75. Livak KJ, Schmittgen TD. Analysis of relative gene expression data using real-time quantitative PCR and the $2-\Delta \Delta C T$ method. Methods. 2001;25(4): 402-8.

\section{Publisher's Note}

Springer Nature remains neutral with regard to jurisdictional claims in published maps and institutional affiliations.

- fast, convenient online submission

- thorough peer review by experienced researchers in your field

- rapid publication on acceptance

- support for research data, including large and complex data types

- gold Open Access which fosters wider collaboration and increased citations

- maximum visibility for your research: over $100 \mathrm{M}$ website views per year

At BMC, research is always in progress.

Learn more biomedcentral.com/submissions 